\title{
Belgeo
}

Revue belge de géographie

\section{Dynamique du couvert végétal à la périphérie du Parc W du Burkina Faso}

Vegetation cover dynamic on the periphery of the Parc W of Burkina Faso

\section{R. Noël Gansaonré, Benewindé J.-B. Zoungrana et P. Isidore Yanogo}

\section{(2) OpenEdition}

1 Journals

Édition électronique

URL : http://journals.openedition.org/belgeo/40786

DOI : $10.4000 /$ belgeo.40786

ISSN : 2294-9135

Éditeur :

National Committee of Geography of Belgium, Société Royale Belge de Géographie

Référence électronique

R. Noël Gansaonré, Benewindé J.-B. Zoungrana et P. Isidore Yanogo, « Dynamique du couvert végétal à la périphérie du Parc W du Burkina Faso », Belgeo [En ligne], 1 | 2020, mis en ligne le 12 juin 2020, consulté le 19 janvier 2021. URL : http://journals.openedition.org/belgeo/40786 ; DOl : https://doi.org/ $10.4000 /$ belgeo.40786

Ce document a été généré automatiquement le 19 janvier 2021.

Belgeo est mis à disposition selon les termes de la licence Creative Commons Attribution 4.0 International. 


\title{
Dynamique du couvert végétal à la périphérie du Parc $W$ du Burkina Faso
}

Vegetation cover dynamic on the periphery of the Parc W of Burkina Faso

\author{
R. Noël Gansaonré, Benewindé J.-B. Zoungrana et P. Isidore Yanogo
}

Nous adressons nos remerciements à la Fondation Internationale pour la Science (FIS) pour le financement des travaux de cette recherche. Nos remerciements vont également à l'endroit de toutes les personnes qui ont contribué d'une manière ou d'une autre à l'élaboration de cet article.

\section{Introduction}

1 Les problèmes environnementaux se sont accentués avec la croissance économique et démographique qu'a connue le monde dans la deuxième moitié du $20^{\text {ème }}$ siècle. La régression des espaces naturels a été à l'origine de mouvements mondiaux qui ont conduit à la mise en œuvre de conventions internationales de protection des ressources naturelles. En dépit de toutes ces dispositions, les ressources naturelles se dégradent dangereusement. De nos jours, les aires protégées constituent un des moyens les plus efficaces de préservation de la nature, malgré les fortes pressions qu'elles connaissent. En effet, la disponibilité des ressources dans ces aires protégées provoque des arrivées de migrants, agriculteurs et des éleveurs, à la recherche de pâturage pour le bétail. C'est le cas du Parc W qui connaît une forte pression (Vermeulen, 2004). Selon Avakoudjo et al. (2014), la croissance démographique, l'extension des superficies agricoles et le surpâturage sont les principaux facteurs de la dégradation du couvert végétal à la périphérie du Parc $\mathrm{W}$ du Bénin. À ces facteurs de dégradation, viennent s'ajouter les variations climatiques qui contribuent au renforcement de la dynamique régressive du couvert végétal.

2 Dans les pays de la zone sahélienne, les études réalisées montrent que les maigres ressources naturelles existantes subissent la pression humaine qui favorise une 
dégradation importante du couvert végétal. Ainsi, selon la FAO (2015), entre 1990 et 2015, le Burkina Faso a perdu annuellement 59900 ha, correspondant à une dégradation annuelle d'environ $1 \%$ de son couvert végétal. Dans l'ouest du pays, zone la plus boisée du pays, précisément autour du Parc National des deux Balé, la régression du couvert végétal est estimée à environ 0,2 \% par an entre 1985 et 2015 (Tankoano et al., 2016). Dans l'extrême sud-est du pays, Soulama et al. (2015) ont monté que $30,8 \% \mathrm{du}$ paysage forestier s'est dégradé entre 2001 et 2013. Ces évolutions régressives sont principalement dues à l'extension des superficies agricoles. Ces études qui sont basées sur l'interprétation des images satellitaires montrent en substance que le couvert végétal au Burkina Faso est en dégradation. Autour du Parc $\mathrm{W}$ du Burkina Faso, plusieurs études en rapport avec la dynamique du couvert végétal ont été menées (Sawadogo, 2012 ; Vermeulen, 2004). Ces études se sont focalisées sur quelques villages et s'appuient uniquement sur des données spatiales collectées à l'aide d'un GPS. Quant à l'étude de Sawadogo (2012), elle a porté sur la dynamique du fourrage dans un village périphérique du Parc $\mathrm{W}$ du Burkina Faso, le village de Kotchari. La dynamique d'occupation des terres réalisée dans cette étude a montré une régression du couvert végétal, cependant, elle ne se limite qu'à un terroir villageois. De même, l'étude de Gansaonré (2018) qui a montré une dégradation du couvert végétal n'a porté que sur la commune de Diapaga et ne prend pas en compte l'indice de végétation de différence normalisée (NDVI). D'autres études portant sur la dynamique du couvert végétal se sont essentiellement intéressées à la composition floristique du couvert végétal (Koadima, 2008; Nacoulma, 2012). Ces études ont montré une régression de plusieurs espèces végétales notamment Khaya senegalensis, Boswellia dalzielii, Tamarindus indica, etc. Pourtant, ces ressources sont importantes pour la survie des populations riveraines du Parc W du Burkina Faso au regard des avantages qu'elles y tirent.

Dans la savane ouest-africaine, plusieurs auteurs ont utilisé les images satellitaires pour analyser la dynamique de la végétation (Ouédraogo et al., 2010 ; Houessou et al., 2013 ; Dimobé et al., 2015 ; Zoungrana et al., 2015 ; Gansaonré, 2018). La plupart d'entre eux se sont basés sur la classification des images de différentes périodes pour détecter les changements dans l'occupation et l'utilisation des terres (Houessou et al., 2013 ; Dimobé et al., 2015 ; Soulama et al., 2015 ; Tankoano et al., 2016; Gansaonré, 2018). Cependant, l'usage des indices tels que l'indice de végétation par différence normalisée (NDVI) pour des études locales demeure rare dans la savane de l'Afrique de l'ouest; pourtant l'analyse de la dynamique du NDVI permet de déterminer la tendance évolutive du couvert végétal et son association avec la dynamique de l'occupation des terres donne des informations plus complètes sur l'évolution de la végétation. L'objectif de cette étude est d'analyser la dynamique du couvert végétal du Parc W du Burkina Faso et sa périphérie à travers les images Landsat et NDVI. L'hypothèse de l'étude présume que les images Landsat et NDVI permettent de mettre en exergue la tendance régressive du couvert végétal. Ce travail s'articulera autour de quatre points, dont la présentation de la zone d'étude, les matériels et méthodes, les résultats et la discussion.

\section{Présentation de la zone d'étude}

La zone d'investigation de la présente recherche se situe dans l'extrême est du Burkina Faso, précisément dans la province de la Tapoa, à la frontière avec le Niger et le Bénin. Crée en 1993 suite à la promulgation de la loi nº03/93/ADP du 7 mai 1993 portant 
organisation de l'administration du territoire, la province de la Tapoa regroupe 7 communes rurales et une commune urbaine (Diapaga). La présente étude s'intéresse au Parc W et sa périphérie. Celle-ci est constituée par les communes de Botou, Diapaga, Tansarga et Logobou. La figure 1 présente la localisation de la zone d'étude.

Figure 1. Localisation des communes périphériques du Parc W.

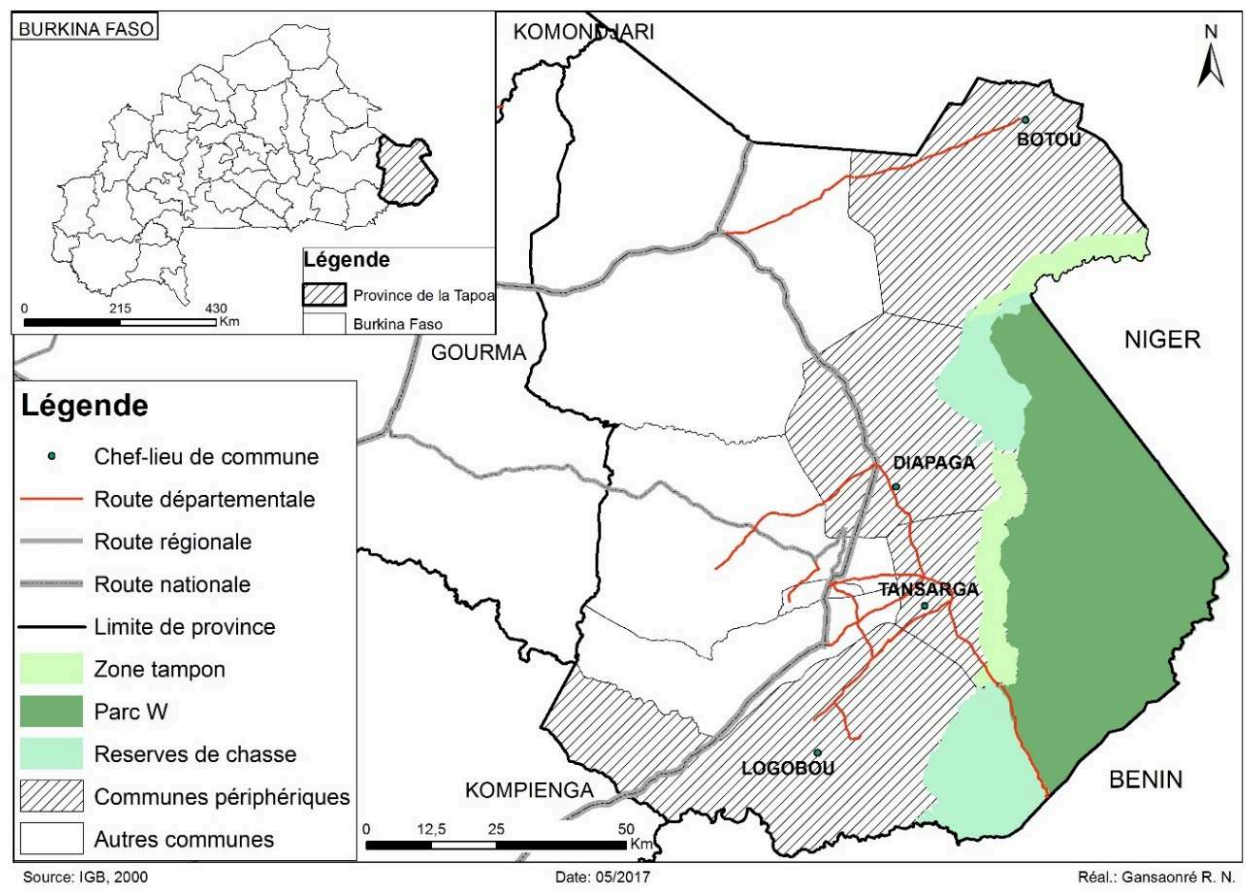

5 Le Parc W a été créé en 1926 par l'administration coloniale française en tant que zone de refuge. Son statut a évolué et devient une réserve totale de faune par arrêté ${ }^{\circ} 2606 /$ SE/F du 14 avril 1953, puis Parc national du W par décret nº6009/SE/T du 4 août 1954 $\mathrm{du}$ gouvernement de l'Afrique Occidentale Française (AOF). Sa mise en réserve a nécessité le déguerpissement de populations, ce qui justifie les différentes formes de revendications autour du parc. Selon Fiasson (1937), quelques hameaux de culture ont été déplacés en 1937 hors du parc à cause de l'influence de l'onchocercose. Les récents classements des zones de chasses ont provoqué des déguerpissements violents (Niembouma et Mouabou en 1989, Antiaga en 1993 et Koyenli en 1998) au point d'être toujours marqués dans les esprits (Kleitz, 2001). Le Parc W est bordé par les concessions de chasse de Kourtiagou, de Tapoa-Djerma et par des zones villageoises d'intérêt cynégétique qui font office de zones tampons. Dans le sud de la commune de Logobou, la concession de chasse de Kourtiagou constitue, avec la zone de chasse de Kokrana, une zone de transition entre le Parc W et le Parc Arly.

La zone d'étude appartient au secteur nord-soudanien suivant le découpage phytogéographique établi par Guinko (1984). Les formations végétales sont dominées par les savanes qui se caractérisent par une dominance de Afzelia africana, Anogeissus leiocarpus, Balanites aegyptiaca, Burkea africana, Crossopteryx febrifuga, Daniella oliveri, Isoberlinia doka, Khaya senegalensis, Kigelia africana, Lannea acida, Mitragyna inermis, Pterocarpus santalinoides, Terminalia avicennioides, Vitellaria paradoxa, etc. Concernant les strates herbacées, elles sont dominées par des graminées et caractérisées par la 
présence des espèces telles que Loudetia togoensis, Vetiveria nigritana, Echinochloa pyramidalis, etc.

7 À cheval sur trois pays, le Niger, le Burkina Faso et le Bénin, le Parc W s'étend sur une superficie de 1045000 ha, dont 235000 ha pour le Parc national W du Burkina Faso. L'intérêt de sa conservation reste très important aussi bien pour la conservation de la faune et de la flore que pour la préservation du patrimoine naturel et biologique dont il dispose. Pour avoir été reconnu comme réserve de la biosphère, patrimoine mondial de l'UNESCO et protégé par la convention Ramsar, il constitue un enjeu mondial important. Malheureusement, le Parc W du Burkina Faso est sous l'effet permanent de la pression animale due à l'arrivée de nombreux transhumants en provenance du Bénin, du Burkina et du Niger (Sawadogo, 2011). Il est également soumis à une importante dynamique foncière liée à la croissance démographique et à la pression agricole (Vermeulen, 2004). Selon Lompo (2002), les superficies des champs de quatorze villages périphériques du Parc W sont passées de 104 ha durant la campagne 1996-1997 à 2038 ha durant la campagne agricole de 2002-2003.

8 Ainsi, le choix de cette zone se justifie aussi bien par la forte pression anthropique due aux mouvements migratoires et à l'augmentation de la population (Vermeulen, 2004) que par les variations climatiques. La zone d'étude appartient à la zone climatique soudano-sahélienne, caractérisée par une longue saison sèche de 7 à 8 mois, s'étalant entre les mois d'octobre et de mai. La saison pluvieuse, plus courte, dure 4 à 5 mois et s'étale entre juin et septembre. La zone d'étude est délimitée par les isohyètes $700 \mathrm{~mm}$ au nord et $1000 \mathrm{~mm}$ au sud. La figure 2 présente l'évolution interannuelle de la pluviométrie de la zone d'étude entre 1950 et 2015.

Figure 2. Variations interannuelles des précipitations de la station de Fada N'Gourma.

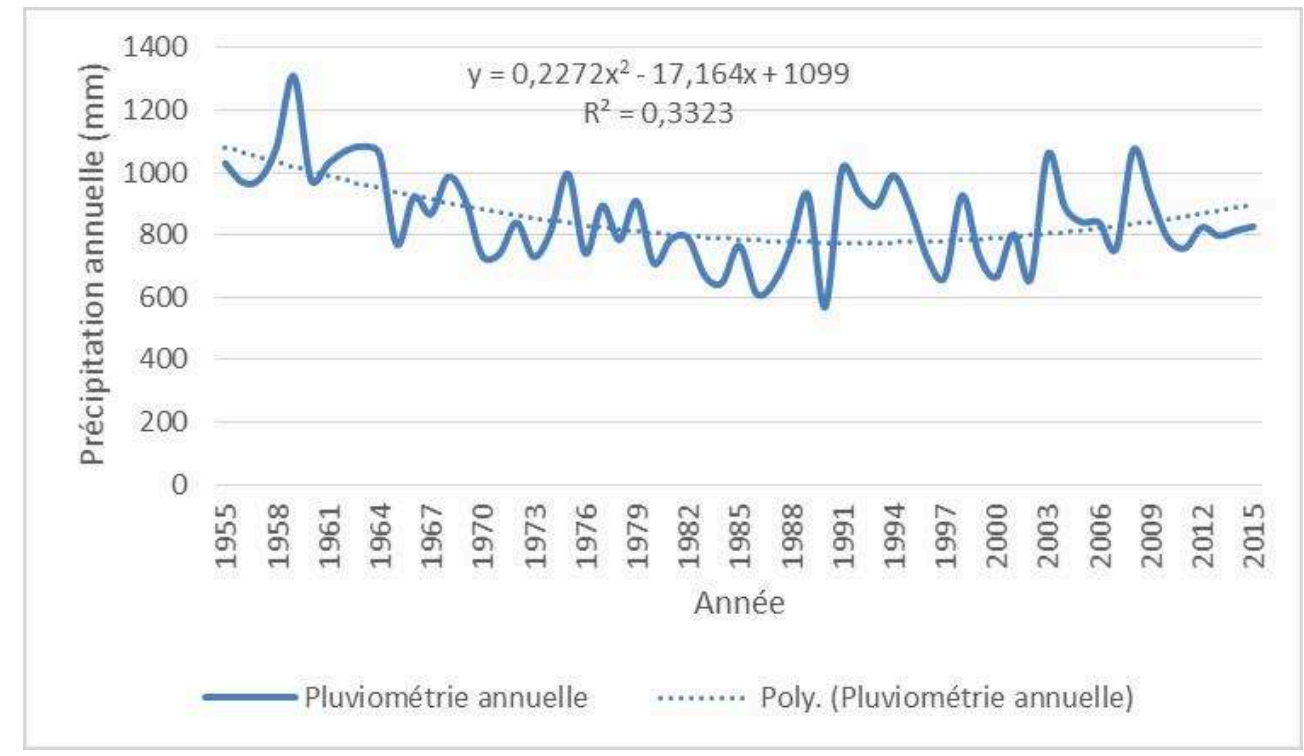

Source : Agence Nationale de Météorologie, 2016

9 L'examen de la figure permet de dégager deux séquences de l'évolution de la pluviométrie. Entre 1950 et 1990, on note une tendance à la diminution de la pluviométrie. À partir de 1990, la courbe montre une tendance à l'augmentation des précipitations. D'autres auteurs, notamment Yanogo (2012) et Hountondji (2008), estiment que la pluviométrie de la zone d'étude a connu une baisse depuis les années 
1950, mais avec une légère reprise à partir des années 1990. Les caractéristiques climatiques de la zone d'étude montrent une variation de la pluviométrie dans le temps et dans l'espace. Pourtant, Zoungrana (2016) montre que la pluviométrie a un impact considérable sur l'évolution de la végétation. Dans ces conditions, la pluviométrie constitue un indicateur important de l'étude de la dynamique du couvert végétal.

10 Le peuplement de la périphérie du Parc $\mathrm{W}$ du Burkina Faso présente une certaine homogénéité ethnique. Elle est essentiellement occupée par les Gourmantché agriculteurs et les Peuls qui ont pour activité principale l'élevage. La population de la zone d'étude a connu un accroissement considérable selon les données des deux derniers recensements de la population (1996 et 2006). Entre 1996 et 2006, le taux d'accroissement global de la population des quatre communes était de $4 \%$ contre 3,1\% pour l'ensemble du pays. Ce taux était de 3,6 \% pour Botou, 3,8 \% pour Logobou, 4,1\% pour Diapaga et $4,6 \%$ pour Tansarga. Dans les quatre communes, la population est passée de 51569 habitants en 1975 à 176098 habitants en 2006, soit une augmentation de $241 \%$ en 31 ans. Elle devrait atteindre un total de 239295 habitants en 2015. Au niveau communal, en 2006, les populations les plus importantes étaient celles de Logobou et de Botou avec respectivement 61418 habitants et 45995 habitants, suivies des communes de Tansarga (36 065 habitants) et de Diapaga (32 620 habitants) (INSD, 1999, 2011).

11 La principale activité économique de la zone d'étude est l'agriculture, qui occupe plus $80 \%$ de la population. L'agriculture est de type familial et est caractérisée par de faibles rendements. Les outils utilisés sont de type traditionnel et ne favorisent pas l'exploitation de vastes superficies. Cependant, on constate une extension progressive des superficies des champs au regard de l'évolution de la démographie. L'agriculture est orientée vers la subsistance. On y produit le sorgho (Sorghum bicolor), le mil (Pennisetum glaucum), le maïs (Zea mays) et le riz (Orysa sativa). Les cultures commerciales sont: l'arachide (Arachis hypogea), le niébé (Vigna unguiculata), le sésame (Sesamum indicum), le soja (Glycine max), le voandzou (Vigna subterrunea) et le coton (Gossypium hirsutum). Cette dernière production a connu un véritable essor à partir des années 2000. Son dynamisme est d'autant plus important qu'il constitue l'une des principales menaces des aires protégées (Vermeulen, 2004). Entre 1990 et 2012, les superficies emblavées par le coton dans la province de la Tapoa ont plus que doublé, passant de 44000 ha à 95000 ha, soit une augmentation de 137,5\%. Quant à l'élevage, il est caractérisé par l'importance de la taille des troupeaux qui varie entre 5 et 70 têtes de bovins chez les agropasteurs gourmantché et 10 à plus de 100 têtes chez les éleveurs peuls. Il est également caractérisé par une divagation des animaux dans la nature (Sawadogo, 2012).

\section{Matériels et méthodes}

\section{Données collectées}

Cette étude a nécessité des images TM, ETM+ et OLI de Landsat (30 m de résolution spatiale) de 1984, 1999 et 2015 sans couverture nuageuse, téléchargées sur le site internet de l'USGS (United States Geological Survey). Pour chaque capteur, trois scènes (193/51, 193/53 et 192/51) couvrant la zone d'étude ont été retenues. Les images de 1984 ont été enregistrées en novembre, alors que les images de 1999 et 2015 ont été 
enregistrées dans le mois d'octobre. Cette période post saison pluvieuse est favorable à une bonne discrimination des unités d'occupation des terres dans la zone soudanosahélienne (Zoungrana et al., 2015). Le choix de ces images vise également à mieux appréhender les changements d'occupation des terres qui sont intervenus à la périphérie du Parc $\mathrm{W}$ ces trente dernières années.

13 En plus des images Landsat, des images NDVI du capteur MODIS (spectroradiomètre imageur à résolution moyenne) de 2001 à 2015 ont été collectées. Le choix de cette période s'explique par le fait que les images NDVI MODIS ne sont disponibles qu'à partir de mars 2000. En effet, MODIS fournit, depuis mars 2000, des informations hebdomadaires. Cependant, des synthèses d'une fréquence de 16 jours sont disponibles. Les données NDVI utilisées dans le cadre de cette étude proviennent du Satellite Terra. Ce choix s'explique par le fait que ce satellite, en balayant la surface terrestre, passe de l'hémisphère nord à l'hémisphère sud dans la matinée, ce qui lui permet de bénéficier d'une bonne réflectance. Au total, 345 images pour les 15 ans d'étude, soit 23 images couvrant toute l'année, de résolution spatiale de $250 \mathrm{~m}$, ont été téléchargées.

Il convient aussi de noter que pour les besoins de la classification des images, des points de références ont été collectés sur le terrain à l'aide d'un GPS pour faciliter le choix des sites d'entraînement. Des informations empiriques ont également été collectées auprès des populations locales afin d'aider pour la classification des images de 1984 et 1999.

\section{Traitement des données}

\section{Traitement des images Landsat}

Les images Landsat, déjà géométriquement corrigées, ont subi des prétraitements radiométriques et atmosphériques qui ont permis de corriger les effets de l'atmosphère et des angles de prise de vue sur la qualité des images.

Le traitement des images Landsat a été réalisé à travers le logiciel ENVI. Une mosaïque d'images a été réalisée à l'aide de l'outil "Mosaicking" avec les différentes scènes (193/51, 193/53 et 192/51) de Landsat couvrant la zone d'étude. Des compositions colorées rehaussées Proche infrarouge/Rouge/Vert ont été utilisées. En s'appuyant sur la nomenclature nationale de la base de données d'occupation des terres élaborée par l'Institut Géographique du Burkina en 2002, les unités d'occupation identifiées dans la zone d'étude sont : la savane boisée (SB), la savane arborée (SAB), la savane arbustive (SAR), les champs ( $\mathrm{CH}$ ), les plans d'eau (PE) et les sols nus (SN)/affleurements rocheux (AF). La discrimination des unités d'occupation et le choix des sites d'entraînement se sont appuyés sur les points de référence collectés sur le terrain. L'algorithme maximum de vraisemblance a été utilisé pour la classification. La précision de la classification a été évaluée à travers l'indice de Kappa.

\section{Traitement des images NDVI}

Les images NDVI ont fait l'objet de prétraitement sur le logiciel Tiseg; ce dernier a permis de corriger les pixels invalides et sans information par interpolation linéaire. Les images ont par la suite été découpées suivant la taille de la zone d'étude. Initialement projetées dans un référentiel sinusoïdal, les images ont été re-projetées dans le système de projection WGS 84 zone 31 correspondant à la zone d'étude. Une série temporelle du NDVI moyen annuel a été calculée pour la période 2001-2015. Cette 
opération a été effectuée afin de disposer de valeurs moyennes du NDVI à partir desquelles les tendances seront calculées.

\section{Analyse des données}

\section{Analyse de la dynamique d'occupation des terres}

L'analyse de la dynamique de l'occupation des terres s'est basée sur les statistiques produites à partir des résultats du traitement des images satellitaires. Les superficies des différentes unités calculées pour chaque année ont permis d'établir le taux d'évolution des unités d'occupation. Ainsi, la superficie d'une unité d'occupation est en croissance si le taux est positif et en réduction lorsque le taux est négatif. L'évolution du couvert végétal a été élaborée dans la perspective d'appréhender la répartition spatiale de la dynamique du couvert végétal.

L'analyse de l'évolution de la végétation a été faite à travers la détection des changements. Le passage d'une unité d'occupation à une autre peut laisser entrevoir une amélioration ou une dégradation. Les unités d'occupation ont été pondérées de 1 à 5 en fonction de l'importance et la densité du couvert végétal (tableau 1). Ainsi, pour une bonne appréciation de l'évolution du couvert végétal, le chiffre 5 a été affecté à l'unité d'occupation la plus densément boisée, la savane boisée et le chiffre 1 à l'unité la plus faiblement boisée, les sols nus et affleurements rocheux.

Tableau 1. Pondération et évaluation de l'évolution du couvert végétal.

\begin{tabular}{|c|c|c|c|}
\hline Unité d'occupation & Pondération & $\begin{array}{l}\text { Gradient } \\
\text { d'amélioration }\end{array}$ & $\begin{array}{ll}\text { Gradient } & \text { de } \\
\text { dégradation } & \end{array}$ \\
\hline Savane boisée & 5 & \multirow[t]{5}{*}{4} & \multirow{5}{*}{$\nabla$} \\
\hline Savane arborée & 4 & & \\
\hline Savane arbustive & 3 & & \\
\hline Champ & 2 & & \\
\hline Sol nu et plan d'eau & 1 & & \\
\hline
\end{tabular}

Source : adapté de Hountondji (2008)

La détection des changements a été faite par le calcul de la variation des valeurs pondérées de chaque pixel à travers le logiciel Arc GIS 10.1 et à l'aide de l'outil « combine» selon la formule suivante (Hountondji, 2008) :

$$
\Delta(p)=\left[V(p)_{2015}-V(p)_{1984}\right]
$$

Avec $\Delta(p)$ : variation du compte numérique du pixel entre 1984 et $2015 . \mathrm{V}(p)_{2015}$ : valeur pondérée du pixel en 1984. $\mathrm{V}(p)_{2014}$ : valeur pondérée du pixel en 2015. 
Les résultats obtenus à l'issue des calculs permettent de dégager le sens d'évolution du couvert végétal du pixel concerné :

- Si $\Delta(p) \geq 3$, alors il y a une forte amélioration du couvert végétal ;

- Si $\Delta(p)=2$, alors il y a une amélioration modérée du couvert végétal ;

- Si $\Delta(p)=1$, alors il y a une faible amélioration du couvert végétal ;

- Si $\Delta(p)=0$, alors l'espace correspondant connaît une stabilité ;

- Si $\Delta(p)=-1$, alors il y a une faible dégradation du couvert végétal ;

- Si $\Delta(p)=-2$ alors il y a une dégradation modérée du couvert végétal ;

- Si $\Delta(p) \leq-3$, alors il y a une forte dégradation du couvert végétal.

Les résultats obtenus de cette opération sont présentés sous forme de cartes.

\section{Analyse de la tendance de la végétation}

\section{Détermination de la tendance du NDVI}

Le test de Mann-Kendall est un test non paramétrique qui est généralement utilisé pour la détection des tendances de données environnementales (Smith, 2000). Il évalue si les valeurs de chaque pixel tendent à croître ou à décroître dans le temps à travers une forme d'analyse des tendances de régression monotone (Donald et al., 2011).

Dans le cadre de cette étude, le test de tendance de Mann-Kendall a été appliqué à la série temporelle du NDVI moyen annuel. L'analyse de la tendance de Mann-Kendall, réalisée à travers le logiciel Idrisi Selva 17, s'établit à deux niveaux.

\section{Détermination du coefficient de corrélation (S) de Mann-Kendall}

Le test de Mann-Kendall analyse le signe de la différence entre les dernières valeurs mesurées et les valeurs mesurées précédemment à travers la fonction. Chaque valeur mesurée est en effet comparée à toutes les valeurs mesurées précédemment (Donald et al., 2011). Le coefficient de corrélation (S) met en exergue la tendance d'évolution de l'indice de végétation. Il est établi selon l'équation ci-dessous :

$$
\begin{aligned}
& S=\sum_{i=1}^{n-1} \sum_{j=n+1}^{n} \text { signe }\left(x_{i}-x_{j}\right) \\
& \text { signe }\left(x_{i}-x_{j}\right)=\left\{\begin{array}{cc}
-1, & \text { si } x_{i}-x_{j}<0 \\
0, & \text { si } x_{i}-x_{j}=0 \\
1, & \text { si } x_{i}-x_{j}>0
\end{array}\right.
\end{aligned}
$$

Où $n$ est la longueur de la série de données étudiées, $x_{i}$ et $x_{j}$, les observations faites à la date $i$ et $j$ respectivement. Pour tout $i=n j=n-1$.

La détermination du taux de tendance de Mann-Kendall $(\tau)$ s'écrit alors comme suit :

$$
\tau=\frac{2 s}{n(n-1)}
$$


avec $s$ le coefficient de corrélation de Mann-Kendall et $n$ la longueur de la série de données étudiées.

En définitive, la valeur du taux de tendance varie entre -1 et 1 en fonction de l'importance de la variation de l'indice de végétation. Ainsi, pour cette étude, il a été retenu que le coefficient de corrélation est positif et important si $\tau \geq 0,5$. Il est négatif et important si $\tau \leq-0,5$. Les valeurs du taux, comprises entre ] $-0,5 ; 0,5$ [, ont une évolution moins importante.

Le mécanisme de calcul est tel que lorsque la valeur de $S$ est un nombre positif important (ou négatif), les valeurs suivantes mesurées ont tendance à être plus grandes (ou plus petites) que les valeurs antérieures et une tendance à la hausse (ou à la baisse) est indiquée. Dans ce cas, l'hypothèse nulle $H_{0}$ est rejetée au profit de l'hypothèse $H_{1}$. Lorsque la valeur absolue de $S$ est faible, aucune tendance n'est indiquée et l'hypothèse nulle $H_{0}$ est acceptée (Donald et al., 2011).

\section{Estimation de la signification du taux de corrélation}

Pour apprécier la tendance évolutive de chaque pixel, le taux de signification de MannKendall a été calculé. Ainsi, la signification de la pente est estimée en utilisant des informations contextuelles de premier ordre. L'évaluation de la signification de la pente s'écrit comme suit :

$$
\begin{aligned}
& Z=\left\{\begin{array}{l}
\frac{S-1}{\sqrt{\operatorname{Var}(S)}} \text { si } S>0 \\
0 \quad \text { si } S=0 \\
\frac{S+1}{\sqrt{\operatorname{Var}(S)}} \text { si } S<0
\end{array}\right. \\
& p=2[1-\phi(|Z|)] \\
& \phi(|Z|)=\frac{2}{\sqrt{\pi}} \int_{0}^{|Z|} e^{-t^{2}} d t
\end{aligned}
$$

Le résultat du test de tendance de Mann-Kendall ainsi mis en œuvre est une image dont les pixels sont affectés de valeurs relatives. Une valeur positive indique une tendance à la hausse tandis qu'une valeur négative indique une tendance à la baisse. La probabilité (p) représente la mesure de l'évidence pour rejeter l'hypothèse nulle. L'interprétation des résultats obtenus permet d'établir le postulat selon lequel pour tout $p \leq 0,05$, le pixel correspondant à un niveau de variation important (Fratianni et Acquaotta, 2010).

35 La méthodologie mise en œuvre a permis d'atteindre un certain nombre de résultats présentés dans le point suivant. 


\section{Résultats}

\section{Cartographie de la dynamique d'occupation des terres}

La classification des images satellitaires de Landsat a produit une précision globale acceptable. En effet, les résultats fournissent des Kappa de $81 \%, 87 \%$ et $92 \%$ respectivement pour les images de 1984, 1999 et 2015. Les résultats obtenus de cette classification peuvent donc être utilisés de façon judicieuse (Pontius, 2000). La figure 3 présente la dynamique d'occupation des terres du Parc W et sa périphérie.

Figure 3. Dynamique d'occupation des terres du Parc W et sa périphérie de 1984 à 2015.

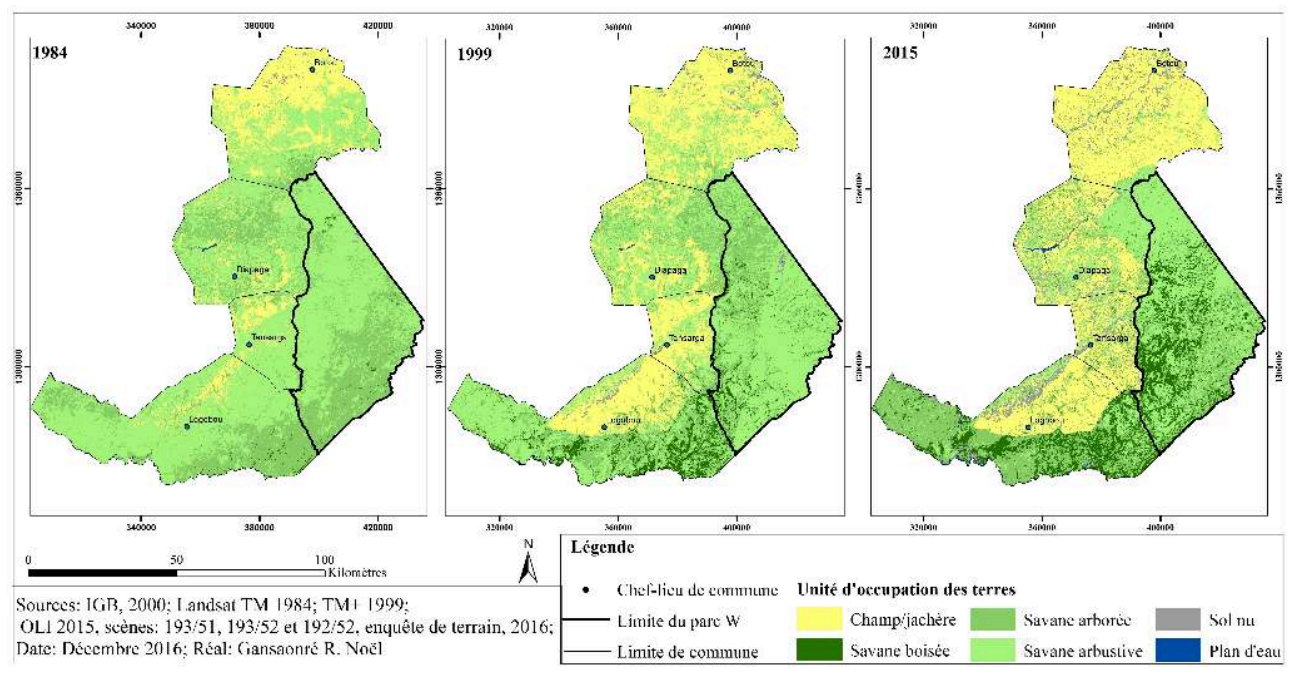

La cartographie de la dynamique d'occupation des terres indique une amélioration du couvert végétal dans le Parc W. Par contre, dans la zone périphérique au parc, on enregistre une dégradation du couvert végétal au profit des superficies agricoles et des zones nues/affleurements rocheux. La savane arbustive, particulièrement, s'est dégradée pour laisser place aux champs (tableau 2).

Tableau 2. Variation des unités d'occupation des terres entre 1984, 1999 et 2015 des zones hors Parc W.

\begin{tabular}{|c|c|c|c|c|c|c|c|c|c|}
\hline \multirow{2}{*}{$\begin{array}{c}\text { Unité } \\
\text { d'occupation }\end{array}$} & \multicolumn{2}{|c|}{1984} & \multicolumn{2}{|c|}{1999} & \multicolumn{2}{c|}{2015} & \multirow{2}{*}{$\begin{array}{c}\text { changement } \\
\text { (1984-2015) }\end{array}$} & $\begin{array}{c}\text { taux } \\
\text { d'évolution } \\
\text { (\%) }\end{array}$ & $\begin{array}{c}\text { taux d'évolution } \\
\text { moyen annuel (\%) }\end{array}$ \\
\cline { 2 - 11 } & Sup. (ha) & $\%$ & Sup. (ha) & $\%$ & Sup. (ha) & $\%$ & & 129,6 & 2,7 \\
\hline CH & 127731 & 20,2 & 257022 & 40,6 & 293245 & 46,4 & 165514 & 3031,4 & 11,8 \\
\hline SB & 1688,9 & 0,3 & 31320,4 & 5,0 & 52885,8 & 8,4 & 51196,9 & 14,4 & 0,4 \\
\hline SAR & 82991,3 & 13,1 & 43054,4 & 6,8 & 94933,3 & 15,0 & 11942 & 14,4 & $-3,3$ \\
\hline SAB & 416292 & 65,8 & 290370 & 45,9 & 144845,2 & 22,9 & $-271446,8$ & $-65,2$ & 3,0 \\
\hline PE & 952,4 & 0,2 & 686,4 & 0,1 & 2350,3 & 0,4 & 1397,9 & 146,8 & \\
\hline SN/AF & 2711,8 & 0,4 & 9914,2 & 1,6 & 44107,8 & 7,0 & 41396 & 1526,5 & 9,4 \\
\hline Total & 632367,4 & 100,0 & 632367,4 & 100,0 & 632367,4 & 100,0 & & & \\
\hline
\end{tabular}

CH : Champ ; sB : Savane boisée ; SAR : Savane arborée ; SAB : Savane arbustive ; PE : Plan d'eau ; SN Sol nu; AF : Affleurement rocheux.

Source : traitement d'images Landsat TM 1984, ETM+ 1999 et OLI 2015 ; scènes 193/51, 193/53 et $192 / 51$ 
Tableau 3. Variation des unités d'occupation des terres entre 1984, 1999 et 2015 du Parc W.

\begin{tabular}{|c|c|c|c|c|c|c|c|c|c|}
\hline \multirow{2}{*}{$\begin{array}{c}\text { Unité } \\
\text { d'occupation }\end{array}$} & \multicolumn{2}{|c|}{1984} & \multicolumn{2}{c|}{1999} & \multicolumn{2}{c|}{2015} & \multirow{2}{*}{$\begin{array}{c}\text { taux } \\
\text { changement } \\
(1984-2015)\end{array}$} & $\begin{array}{c}\text { t'évolution (\%) } \\
\text { taux d'évolution } \\
\text { moyen annuel (\%) }\end{array}$ \\
\cline { 2 - 11 } & Sup. (ha) & $\%$ & Sup. (ha) & $\%$ & Sup. (ha) & $\%$ & & & \\
\hline SB & 1517,1 & 0,6 & 12354,6 & 5,3 & 51448,2 & 22,0 & 49931,1 & 3291,2 & 12,0 \\
\hline SAR & 142314,7 & 60,8 & 147588,6 & 63,0 & 114427,7 & 48,9 & -27887 & $-19,6$ & $-0,7$ \\
\hline SAB & 89693 & 38,3 & 73239 & 31,3 & 67288,8 & 28,7 & $-22404,2$ & $-25,0$ & $-0,9$ \\
\hline PE & 0,6 & 0,0 & 6,6 & 0,0 & 75,7 & 0,0 & 75,1 & 12516,7 & 16,9 \\
\hline SN/AF & 668,2 & 0,3 & 1004,8 & 0,4 & 953,2 & 0,4 & 285 & 42,7 & 1,2 \\
\hline Total & 234193,6 & 100,0 & 234193,6 & 100,0 & 234193,6 & 100,0 & & & \\
\hline
\end{tabular}

SB : Savane boisée ; SAR : Savane arborée ; SAB : Savane arbustive ; PE : Plan d'eau ; SN : Sol nu ; AF Affleurement rocheux

Source : traitement d'images Landsat TM 1984, ETM+ 1999 et OLI 2015 ; scènes 193/51, 193/53 et 192/51

L'examen du tableau montre d'importantes variations de l'occupation des terres. La savane arbustive a perdu $19,9 \%$ et $23 \%$ de sa superficie respectivement entre 1984-1999 et 1999-2015, soit une réduction de 65,2 \% de sa superficie en 31 années. Il en résulte que chaque année, la savane arbustive perd environ 3,3\% de sa superficie. Cette réduction de la superficie de la savane arbustive s'explique par une augmentation de la superficie des champs. Elle est passée de 20,2 à 40,6 \% entre 1984-1999, puis à $46,4 \%$ en 2015. Ainsi, entre 1984 et 2015, la superficie des champs a connu une croissance moyenne annuelle de 2,7\%. L'augmentation des superficies des champs s'explique essentiellement par l'augmentation de la population et les faibles rendements de l'activité agricole. De même, la savane boisée et la savane arborée ont connu des augmentations de leur superficie. L'augmentation remarquable de la superficie de la savane boisée, dont la superficie est passée de $0,3 \%$ à 8,4 \%, s'expliquerait par la mise en réserve des zones de chasse de Tapoa-Djerma, de Kourtiagou et de Kokrana. On note également une augmentation de la superficie des plans d'eau et des sols nus/ affleurements rocheux, qui ont connu des variations respectives de $146,8 \%$ et $1526,5 \%$ entre 1984 et 2015. En effet, l'accroissement des superficies des plans d'eau est dû à l'aménagement du barrage de Diapaga, tandis que l'évolution des sols nus/ affleurements rocheux relève de la dégradation due aux exploitations agricoles.

$\mathrm{Au}$ niveau du Parc W, le tableau 3 montre une augmentation des superficies de la savane boisée. Entre 1984 et 1999, la savane boisée est passée de $0,6 \%$ à 5,3\%, puis à $22 \%$ en 2015. Cette importante évolution s'explique aussi bien par les efforts de mise en défens de l'aire protégée, mais aussi par l'amélioration des conditions climatiques qui a favorisé le développement de la végétation du parc. L'augmentation des superficies de la savane boisée, qui suppose une densification et une croissance des arbres, s'est faite au détriment de la savane arbustive et de la savane arborée. L'importante augmentation des superficies des plans d'eau s'expliquerait par les aménagements de retenues d'eau dans le parc pour l'abreuvement de la faune sauvage. Les tableaux 4 et 5 montrent comment les unités d'occupation ont évolué à la périphérie du parc sur les deux périodes de l'étude. 
Tableau 4. Matrice de transition des unités d'occupation des terres hors parc entre 1984 et 1999 en pourcentage.

\begin{tabular}{|c|c|c|c|c|c|c|c|c|c|}
\hline \multicolumn{10}{|c|}{1984} \\
\hline \multirow{8}{*}{1999} & & $\mathrm{CH}$ & SB & SAR & $\mathrm{SAB}$ & PE & $\mathrm{SA} / \mathrm{AF}$ & $\begin{array}{c}\text { Proportion } \\
\text { en } 1999\end{array}$ & Variation \\
\hline & $\mathrm{CH}$ & 16,1 & 0,0 & 3,4 & 20,9 & 0,0 & 0,3 & 40,6 & 20,4 \\
\hline & SB & 0,0 & 0,2 & 3,3 & 1,5 & 0,0 & 0,0 & 4,9 & 4,7 \\
\hline & SAR & 0,4 & 0,0 & 8,7 & 9,5 & 0,0 & 0,0 & 18,6 & $-2,9$ \\
\hline & SAB & 2,8 & 0,0 & 6,1 & 25,2 & 0,0 & 0,1 & 34,2 & $-23,4$ \\
\hline & PE & 0,0 & 0,0 & 0,0 & 0,0 & 0,0 & 0,0 & 0,1 & 0,0 \\
\hline & $\mathrm{SA} / \mathrm{AF}$ & 0,9 & 0,0 & 0,0 & 0,6 & 0,0 & 0,1 & 1,6 & 1,1 \\
\hline & $\begin{array}{c}\text { Proportion } \\
\text { en } 1984\end{array}$ & 20,2 & 0,3 & 21,4 & 57,6 & 0,1 & 0,4 & 100,0 & \\
\hline
\end{tabular}

CH : Champ ; SB : Savane boisée ; SAR : Savane arborée ; SAB : Savane arbustive ; PE : Plan d'eau ; SN Sol nu; AF : Affleurement rocheux

Source : traitement d'images Landsat TM 1984 et ETM+ 1999 ; scènes 193/51, 193/53 et 192/51

Tableau 5. Matrice de transition des unités d'occupation des terres hors parc entre 1999 et 2015 en pourcentage.

\begin{tabular}{|c|c|c|c|c|c|c|c|c|c|}
\hline \multicolumn{10}{|c|}{1999} \\
\hline & & $\mathrm{CH}$ & SB & SAR & $\mathrm{SAB}$ & $\mathrm{PE}$ & SN/AF & $\begin{array}{l}\text { Proportion } \\
\text { en } 2015\end{array}$ & Variation \\
\hline \multirow{7}{*}{2015} & $\mathrm{CH}$ & 31,0 & 0,1 & 3,5 & 11,0 & 0,0 & 0,7 & 46,3 & 5,6 \\
\hline & SB & 0,3 & 3,6 & 3,7 & 0,8 & 0,0 & 0,0 & 8,4 & 3,4 \\
\hline & SAR & 4,0 & 1,2 & 7,9 & 9,4 & 0,0 & 0,0 & 22,5 & 3,9 \\
\hline & $\mathrm{SAB}$ & 2,1 & 0,1 & 3,2 & 10,1 & 0,0 & 0,1 & 15,5 & $-18,7$ \\
\hline & $\mathrm{PE}$ & 0,1 & 0,0 & 0,0 & 0,2 & 0,1 & 0,0 & 0,4 & 0,3 \\
\hline & $\mathrm{SN} / \mathrm{AF}$ & 3,1 & 0,0 & 0,3 & 2,7 & 0,0 & 0,8 & 7,0 & 5,4 \\
\hline & $\begin{array}{l}\text { Propor } \\
\text { en } 1999\end{array}$ & 40,6 & 4,9 & 18,6 & 34,2 & 0,1 & 1,6 & 100,0 & \\
\hline
\end{tabular}

CH : Champ ; sB : Savane boisée ; SAR : Savane arborée ; SAB : Savane arbustive ; PE : Plan d'eau ; SN Sol nu ; AF : Affleurement rocheux

Source : traitement d'images Landsat ETM+ 1999 et OLI 2015 ; scènes 193/51, 193/53 et 192/51

Le tableau 4 montre qu'entre 1984 et 1999, 20,1\% de la savane arbustive et 3,4\% de la savane arborée se sont converties en champ. Durant cette même période, 9,5\% de la savane arbustive sont devenues de la savane arborée. On note également que 6,1\% de la savane arborée s'est transformée en savane arbustive. Sur la seconde période (tableau 5), les changements sont moins importants relativement à la période précédente. Ainsi, entre 1999 et $2015,11 \%$ et $9,4 \%$ de la savane arbustive se sont converties respectivement en champs et en savane arborée. Le tableau 5 montre également que $4 \%, 3,1 \%$ et $2,1 \%$ des champs se sont convertis respectivement en savane arborée, sol $\mathrm{nu} /$ affleurement rocheux et savane boisée. Ainsi, les résultats montrent que la savane arbustive connaît le plus de changement sur les deux périodes. Cela s'explique non seulement par les sécheresses des années 1983-1984, mais aussi par le fait que la savane arbustive est plus prisée par les populations pour l'aménagement de nouveaux champs. L'augmentation de la superficie des plans d'eau s'explique par les aménagements de 
barrages. Les tableaux 6 et 7 présentent l'évolution des unités d'occupation des terres du Parc W entre 1984-1999 et 1999-2015.

Tableau 6. Matrice de transition des unités d'occupation des terres du Parc W entre 1984 et 1999 en pourcentage.

\begin{tabular}{|c|c|c|c|c|c|c|c|c|}
\hline \multicolumn{9}{|c|}{1984} \\
\hline & & SB & SAR & $\mathrm{SAB}$ & $\mathrm{PE}$ & $\mathrm{SN} / \mathrm{AF}$ & $\begin{array}{l}\text { Proportion } \\
\text { en } 1984\end{array}$ & Variation \\
\hline \multirow{6}{*}{1999} & SB & 0,1 & 3,2 & 2,0 & 0,0 & 0,0 & 5,3 & 4,6 \\
\hline & SAR & 0,2 & 11,5 & 19,4 & 0,0 & 0,0 & 31,3 & $-7,0$ \\
\hline & $\mathrm{SAB}$ & 0,3 & 23,5 & 39,0 & 0,0 & 0,2 & 63,0 & 2,3 \\
\hline & $\mathrm{PE}$ & 0,0 & 0,0 & 0,0 & 0,0 & 0,0 & 0,0 & 0,0 \\
\hline & $\mathrm{SN} / \mathrm{AF}$ & 0,0 & 0,0 & 0,4 & 0,0 & 0,0 & 0,4 & 0,1 \\
\hline & $\begin{array}{l}\text { Proportion } \\
\text { en } 1999\end{array}$ & 0,6 & 38,3 & 60,8 & 0,0 & 0,3 & 100,0 & \\
\hline
\end{tabular}

SB : Savane boisée ; SAR : Savane arborée ; SAB : Savane arbustive ; PE : Plan d'eau ; SN : Sol nu ; AF Affleurement rocheux

Source : traitement d'images Landsat TM 1984, OLI 2015 ; scènes 193/51, 193/53 et 192/51

Tableau 7. Matrice de transition des unités d'occupation des terres du Parc W entre 1999 et 2015 en pourcentage.

\begin{tabular}{|c|c|c|c|c|c|c|c|c|}
\hline \multicolumn{9}{|c|}{1999} \\
\hline & & SB & SAR & $\mathrm{SAB}$ & $\mathrm{PE}$ & $\mathrm{SN} / \mathrm{AF}$ & $\begin{array}{c}\text { Proportion } \\
\text { en } 1999\end{array}$ & Variation \\
\hline \multirow{6}{*}{2015} & SB & 4,0 & 10,3 & 7,7 & 0,0 & 0,0 & 22,0 & 16,7 \\
\hline & SAR & 0,8 & 8,1 & 19,7 & 0,0 & 0,1 & 28,7 & $-2,5$ \\
\hline & $\mathrm{SAB}$ & 0,5 & 12,8 & 35,2 & 0,0 & 0,3 & 48,9 & $-14,2$ \\
\hline & $\mathrm{PE}$ & 0,0 & 0,0 & 0,0 & 0,0 & 0,0 & 0,0 & 0,0 \\
\hline & $\mathrm{SN} / \mathrm{AF}$ & 0,0 & 0,0 & 0,4 & 0,0 & 0,0 & 0,4 & 0,0 \\
\hline & $\begin{array}{l}\text { Proportion } \\
\text { en } 2015\end{array}$ & 5,3 & 31,3 & 63,0 & 0,0 & 0,4 & 100,0 & \\
\hline
\end{tabular}

SB : Savane boisée ; SAR : Savane arborée ; SAB : Savane arbustive ; PE : Plan d'eau ; SN : Sol nu ; AF Affleurement rocheux

Source : traitement d'images Landsat ETM+ 1999, OLI 2015 ; scènes 193/51, 193/53 et 192/51

41 Entre 1984 et 1999, la superficie de la savane arborée a connu une régression, passant de $31,3 \%$ à $28,7 \%$. Cette régression s'est faite au profit de la savane arbustive $(23,5 \%)$ et de la savane boisée (3,2\%). Il convient aussi de souligner d'importantes conversions de la savane arbustive à la savane arborée $(19,4 \%)$ et à la savane boisée (2\%). De 1999 à 2015 , le tableau 7 montre une régression de la savane arbustive $(-14,2 \%)$ et de la savane arborée $(-2,5 \%)$ au profit de la savane boisée. Ainsi, 10,3\% et $12,8 \%$ de la savane arborée se sont transformés respectivement en savane boisée et en savane arbustive. Alors que $19,7 \%$ et $7,7 \%$ de la savane arbustive se sont converties respectivement en savane arborée et savane boisée.

Ces changements montrent une amélioration du couvert végétal du parc contrairement à la zone hors parc. En effet, dans la zone hors parc, le couvert végétal s'est progressivement dégradé au profit des champs. Sur l'ensemble de la période d'étude, les unités de végétation ont connu une régression de leur superficie. Par contre, dans l'aire du parc, on enregistre une amélioration du couvert végétal qui se manifeste par 
une augmentation de la superficie de la savane boisée. La figure 4 qui découle de l'équation 1 permet une meilleure appréciation de l'évolution du couvert végétal.

Figure 4. Tendances évolutives du couvert végétal entre 1984 et 2015.

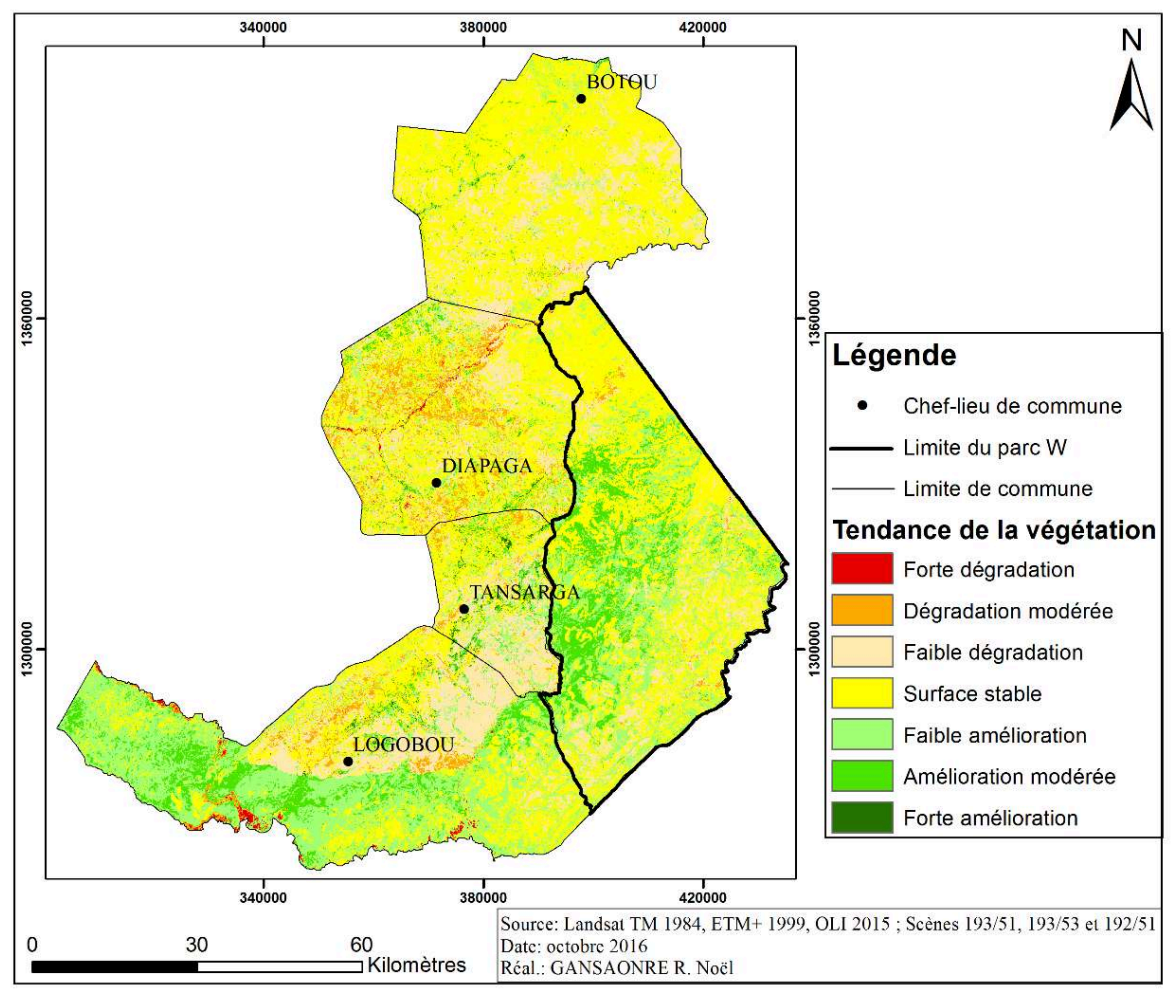

L'examen de la figure 4 montre que les formations végétales ayant connu une évolution progressive sont principalement localisées dans les aires protégées, le Parc W et les zones de chasse de la commune de Logobou. Les champs apparaissent comme des unités stables, puisque $48,6 \%$ des zones stables sont occupées par ceux-ci. Le tableau 8 présente les proportions des tendances évolutives des unités d'occupation des terres du Parc $\mathrm{W}$ et sa périphérie.

Tableau 8. Tendance évolutive du couvert végétal du Parc W et sa périphérie de 1984 à 2015.

\begin{tabular}{|l|c|c|c|c|c|}
\hline Tendance & Parc W & Botou & Diapaga & Tansarga & Logobou \\
\hline Forte dégradation & 0,1 & 0,2 & 1,8 & 0,3 & 1,2 \\
\hline $\begin{array}{l}\text { Dégradation } \\
\text { modérée }\end{array}$ & 0,6 & 5,9 & 15,1 & 11,3 & 5,7 \\
\hline Faible dégradation & 10,6 & 42,1 & 31,4 & 40,7 & 20,8 \\
\hline Stable & 50,8 & 47,7 & 40,4 & 26,8 & 25,2 \\
\hline Faible amélioration & 24,9 & 3,2 & 9,5 & 15,6 & 38 \\
\hline $\begin{array}{l}\text { Amélioration } \\
\text { modérée }\end{array}$ & 13,0 & 0,8 & 1,8 & 5 & 9 \\
\hline Forte amélioration & 0,0 & 0,1 & 0,1 & 0,4 & 0,1 \\
\hline Total & 100 & 100 & 100 & 100 & 100 \\
\hline
\end{tabular}

Source : traitement d'images Landsat TM 1984 et OLI 2015 ; scènes 193/51, 193/53 et 192/51

Le tableau 8 montre que les communes riveraines du Parc W connaissent une dégradation du couvert végétal. On note ainsi que $88,1 \%, 48,3 \%$ et $52,2 \%$ des superficies respectives de Botou, Diapaga et Tansarga sont en dégradation, alors que le 
Parc W et la commune de Logobou connaissent un reverdissement, puisque respectivement $37,9 \%$ et $47,2 \%$ de leurs superficies ont connu un reverdissement entre 1984 et 2015. L'évolution de la végétation dans la commune de Logobou s'explique par l'existence des zones de chasse de Kokrana et de Kourtiagou.

\section{Évolution de l'indice de végétation NDVI entre 2001-2015}

Les figures $5 a$ et $5 b$, résultant du test de Mann-Kendall (cf. équations 4 et 6), montrent respectivement le signe de la tendance évolutive du NDVI et du niveau de signification. Entre 2001 et 2015, 89,32 \% de la zone d'étude ont connu une évolution régressive du NDVI, tandis que $10,67 \%$ ont connu une progression (figure $5 \mathrm{a}$ ). On note également que le niveau de signification $(\mathrm{P}<0.05)$ est plus net au niveau des tendances négatives (figure $5 b$ ) selon les résultats fournis par l'équation 6 . La carte 5 , issue du croisement entre le coefficient de corrélation et le niveau de signification, révèle une forte fréquence de tendance végétale non significative. Cependant, des tendances significatives ont également été observées, et parmi elles, on note une large occurrence de tendances régressives significatives de la végétation $(16,82 \%)$ et une absence de tendance positive significative durant la période 2001-2015.

Figure 5. Tendance de NDVI du Parc W et sa périphérie durant la période 2001-2015 (a) évolution du NDVI ; (b) niveau de signification de l'évolution du NDVI.

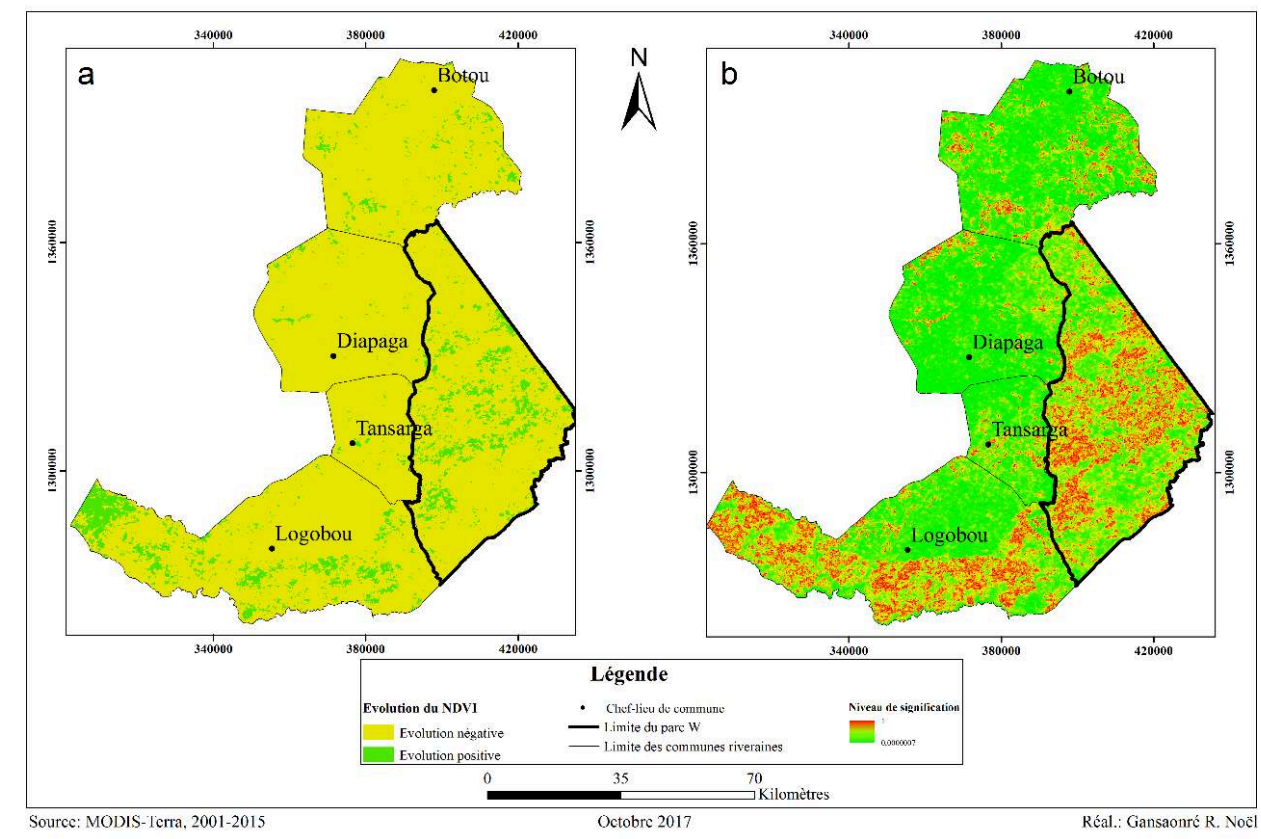

Le croisement des résultats issus de la détermination du taux de corrélation et le niveau de signification de la tendance de l'indice de végétation permet d'obtenir une nouvelle image. Cette image présente le niveau de signification de la tendance évolutive de l'indice de végétation, selon qu'elle soit positive ou négative (figure 6). 
Figure 6. Tendance évolutive de l'indice de végétation du Parc W et sa périphérie.

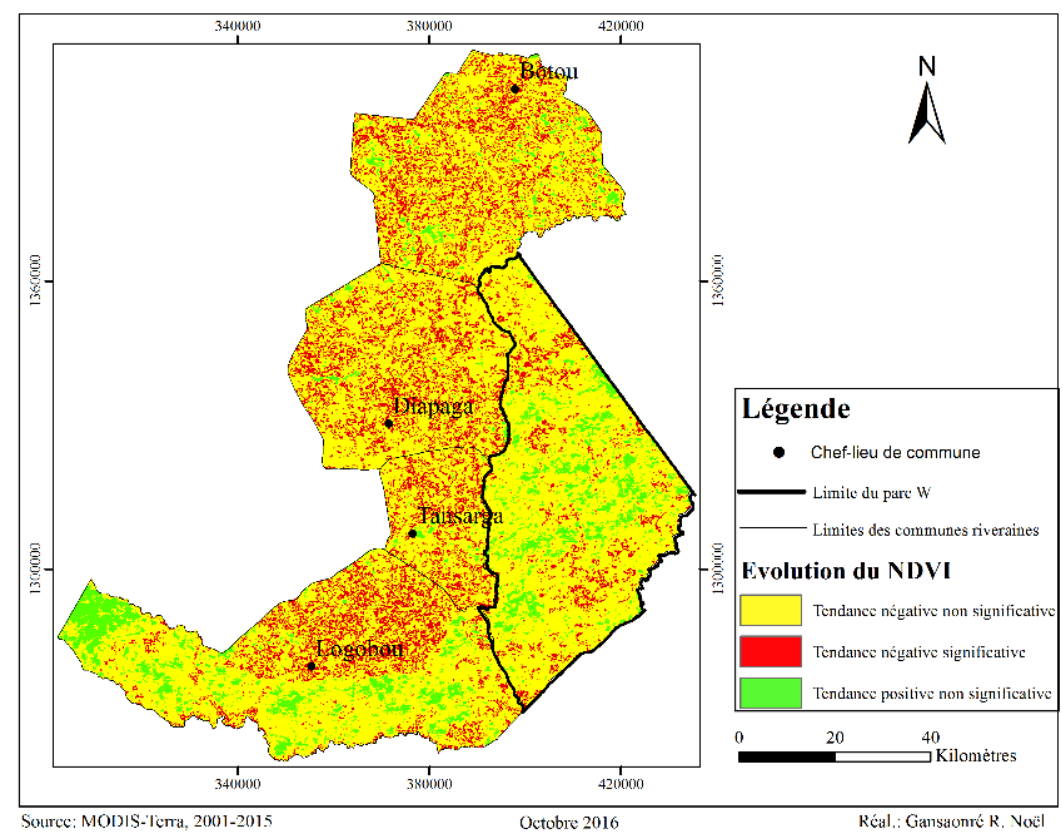

Tableau 9. Tendance évolutive de la végétation par unité d'occupation en pourcentage.

\begin{tabular}{|c|c|c|c|c|}
\hline $\begin{array}{c}\text { Unité } \\
\text { d'occupation }\end{array}$ & $\begin{array}{c}\text { Tendance négative } \\
\text { significative }\end{array}$ & $\begin{array}{c}\text { Tendance négative } \\
\text { non significative }\end{array}$ & $\begin{array}{c}\text { Tendance positive } \\
\text { non significative }\end{array}$ & Total \\
\hline CH & 7,88 & 23,72 & 2,23 & 33,83 \\
\hline SB & 1,08 & 9,00 & 1,95 & 12,04 \\
\hline SAR & 2,95 & 17,71 & 3,50 & 24,16 \\
\hline SAB & 3,72 & 18,14 & 2,62 & 24,48 \\
\hline PE & 0,07 & 0,21 & 0,00 & 0,28 \\
\hline SN/AF & 1,11 & 3,71 & 0,37 & 5,2 \\
\hline Total & 16,82 & 72,50 & 10,67 & 100,0 \\
\hline
\end{tabular}

CH : Champ ; SB : Savane boisée ; SAR : Savane arborée ; SAB : Savane arbustive ; PE : Plan d'eau ; SN Sol nu; AF : Affleurement rocheux

Source : traitement Modis-Terra, 2001-2015 ; Landsat, 1984 et 2015, scènes 193/51, 193/52 et 192/51

47 Le tableau 9 montre que les champs $(7,88 \%)$ et les savanes arbustives $(3,72 \%)$ sont les unités d'occupation les plus affectées par les tendances négatives significatives du NDVI. Cela pourrait s'expliquer par les actions de l'homme qui influencent régulièrement la végétation et les variations pluviométriques. Les tendances négatives non significatives sont également plus importantes au niveau des champs $(23,72 \%)$ et de la savane arbustive $(18,14 \%)$. Tandis que les tendances positives non significatives s'expriment davantage au sein de la savane arborée (3,5\%). 


\section{Discussion}

48 La dynamique de l'occupation des terres a montré une tendance à la dégradation du couvert végétal dans la périphérie du Parc W, puisque les communes de Botou, Diapaga et Tansarga connaissent une importante dégradation du couvert végétal sur plus de $40 \%$ de leur superficie. Dans la zone périphérique du Parc W, entre 1984 et 1999, la superficie des champs a progressé de plus de $20 \%$. En 2015, la superficie de cette unité d'occupation a plus que doublé. Cette évolution se fait au détriment du couvert végétal. Ainsi, la savane arbustive a connu une régression sur les deux périodes de l'étude. Par contre, on relève, à la fin des deux périodes, une augmentation des superficies de la savane boisée et de la savane arborée. Ces augmentations sont insignifiantes face à l'évolution régressive de la savane arbustive, qui semble être la couche de végétation la plus prisée par la population. L'augmentation de la population, les mouvements migratoires et la politique agricole instaurée autour des années 2000 ont alors favorisé l'expansion de la culture cotonnière, ce qui explique l'importance de l'évolution des superficies agricoles et la réduction des superficies du couvert végétal. Toutefois, l'augmentation des superficies de la savane boisée et arborée pourrait s'expliquer par l'intervention des ONG, des acteurs communaux et des structures techniques de l'État qui ont permis l'aménagement d'aires protégées communautaires dénommées Zones Villageoises d'Intérêt Cynégétique (ZOVIC) (Gansaonré et al., inpress). Ces espaces communautaires ont parfois joué le rôle de zone tampon entre les aires d'habitation et le Parc W, tout comme les zones de chasse contiguës au Parc W.

Les résultats ont montré que le couvert végétal du Parc W s'est nettement amélioré entre 1984 et 2015, puisque plusieurs projets de préservation y ont été mis en œuvre. Ces résultats sont conformes à plusieurs études menées dans la zone d'étude (Vermeulen, 2004 ; Nacoulma, 2012). Dans l'ensemble, on constate une diminution de la superficie des savanes arborée et arbustive au profit de la savane boisée. Contrairement au Parc National des deux Balé où la pression anthropique a abouti à une colonisation de l'aire protégée par les agriculteurs (Tankoano, 2016), les acteurs intervenant autour du Parc $\mathrm{W}$ ont jusque-là pu contenir l'évolution du front agricole en direction du parc.

Les résultats obtenus montrant une importante dégradation du couvert végétal dans la périphérie du parc et une amélioration à l'intérieur sont similaires aux conclusions de Soulama et al. (2015). Les travaux de Tankoano et al. (2016) et Zoungrana et al. (2015) qui ont été réalisés dans la région soudanienne, notamment dans les forêts de Pâ, Dibon, des Deux Balé et leurs périphéries dans le Sud-Ouest burkinabè ont également montré une régression du couvert végétal. Des résultats similaires ont également été obtenus par Dipama (2009) autour du Parc National Tambi Kaboré au Burkina Faso.

51 Concernant la tendance évolutive de la végétation, le test de Mann-Kendall a montré une tendance générale à la régression durant la période 2001-2015. Le croisement entre l'occupation des terres en 2015 et les résultats du test de Mann-Kendall a montré que l'activité humaine constitue la principale cause de la régression significative de l'indice de végétation (NDVI). D'autres facteurs non moins importants que sont les variations climatiques influencent également la dynamique de la végétation, puisqu'elles provoquent le stress de la végétation et une pression sur les ressources. À cet effet, Zoungrana (2016) établit une forte corrélation entre l'évolution du NDVI et la pluviométrie dans le sud-ouest du pays, traduisant un impact important de la variation pluviométrique sur la végétation. De même, Roudier (2012) a montré qu'en Afrique de 
l'ouest, les variations climatiques, notamment les fluctuations pluviométriques, ont favorisé l'accroissement des superficies agricoles, donc une dégradation du couvert végétal. Rasmussen et al. (2014) dans leur analyse expliquent que l'évolution régressive du NDVI dans la zone sahélienne du Burkina Faso est à $30 \%$ due à la variation de la pluviométrie; les autres causes pouvant être d'origine naturelle ou anthropique. Ils révèlent par ailleurs l'importance de la dynamique d'occupation des terres principalement influencée par la croissance des exploitations agropastorales. Cette argumentation concorde avec les résultats de cette étude spatio-temporelle de l'évolution du NDVI. En effet, la périphérie du Parc W présente une tendance générale à la dégradation du couvert végétal. Des cas d'amélioration sont perceptibles dans les aires protégées du W, d'Arly et des concessions de chasse de Kokrana et de Kourtiagou, ce qui laisse entrevoir l'importance de l'action humaine sur la dynamique de l'indice de végétation des communes périphériques du Parc W. Ainsi, Ozer et al. (2010) et Avakoudjo et al. (2014) attribuent la dégradation du couvert végétal à la croissance démographique. En effet, Ozer et al. (2010) estiment que la pluviométrie dans la zone sahélienne s'est nettement améliorée par rapport à la période sèche qu'a connue l'Afrique de l'ouest dans les années 1970 et 1980 . On peut donc déduire de cette analyse que les aires protégées constitueraient un moyen efficace de préservation des écosystèmes et de la biodiversité. C'est pourquoi de nombreuses actions sont mises en œuvre aussi bien niveau national qu'international pour la préservation de ces aires.

L'évolution de l'indice de végétation traduit une disponibilité de moins en moins suffisante de pâturages principalement recherchés dans la savane arbustive. Ainsi, la régression du couvert végétal témoigne également de la réduction de l'activité chlorophyllienne de la végétation qui s'explique, soit par la réduction de la densité du couvert végétal, soit par l'accroissement du stress hydrique, soit par le vieillissement de la végétation (figure 7). 
Figure 7. Faciès d'une savane arbustive sur sol peu profond.

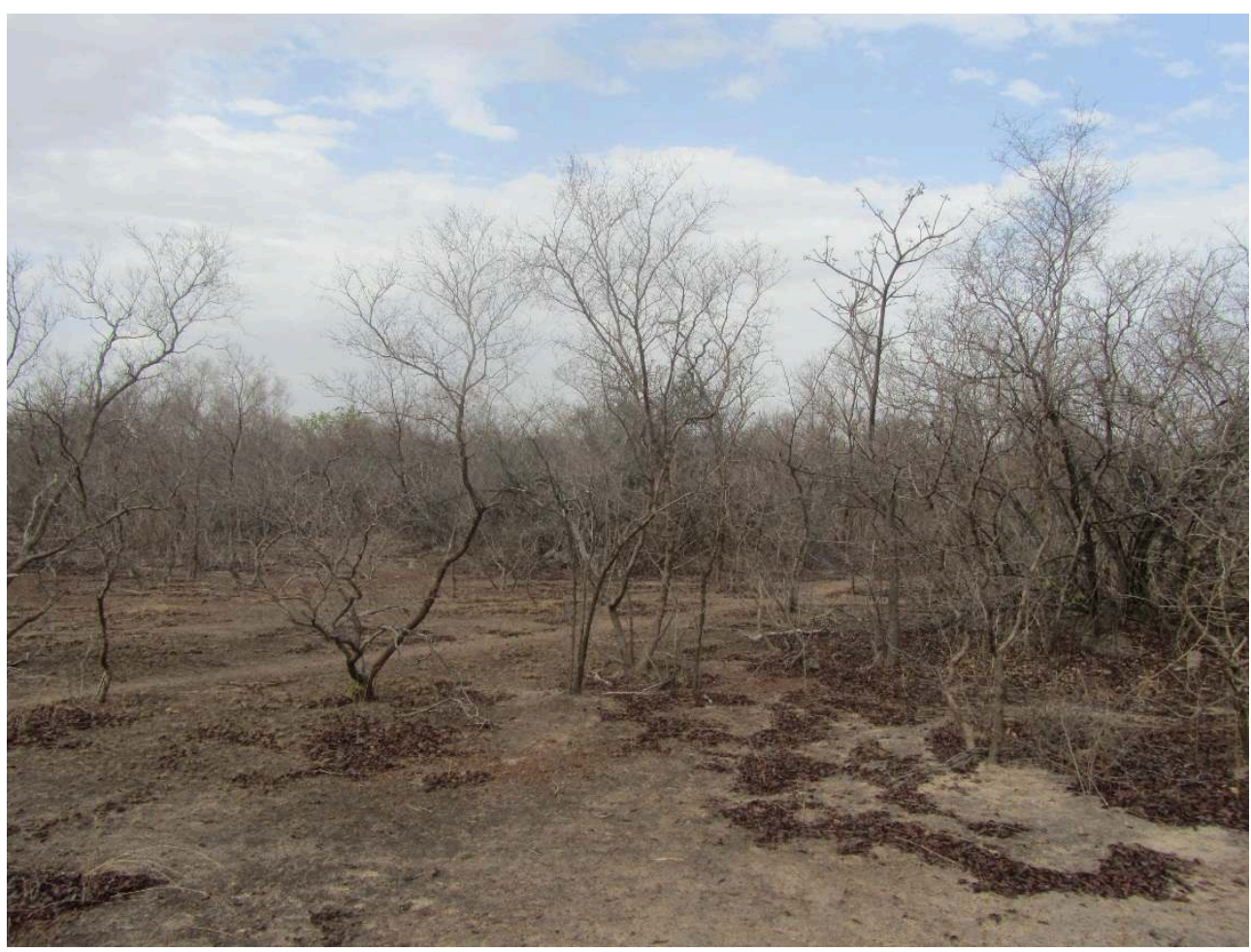

On remarque un assèchement de la végétation traduisant une faible activité chlorophyllienne. Image prise dans le village de Barpoa, commune de Diapaga.

Prise de vue : GANSAONRE R. Noël, enquête de terrain, juin 2016

L'évolution du couvert végétal à la périphérie du Parc W du Burkina Faso s'imbrique dans une dynamique régressive qui se manifeste au niveau national. Dans l'ensemble du pays, plusieurs auteurs ont montré de fortes dégradations du couvert végétal principalement dues à des facteurs anthropiques. Cela s'accompagne de fortes érosions, d'un épuisement des sols et de la réduction de la productivité agropastorale (Emeterio et al., 2013). À la périphérie du Parc W du Bénin et du Niger, des états de dégradation sont aussi notés (Avakoudjo et al., 2014). Ainsi, la dégradation du couvert végétal est un phénomène commun à l'ensemble des pays de l'Afrique subsaharienne. Cette dynamique, qui se manifeste par la dégradation de vastes superficies de végétation, a des implications importantes au niveau mondial. En effet, dans le contexte actuel de changements environnementaux globaux, les formations végétales constituent un moyen d'atténuation par la séquestration de certains gaz à effet de serre, nomment la $\mathrm{CO}_{2}$ (Ciesla, 1997). C'est pour cela que la préservation des formations végétales a été mise au centre des négociations du protocole de Kyoto, notamment la réduction des émissions des gaz à effet de serre due à la déforestation et à la dégradation forestière (REDD+) (Tsayem Demaze, 2009) dans la perspective d'une compensation financière pour la préservation du couvert végétal. Bien que des incertitudes subsistent encore dans le processus de mise en œuvre de la REDD+ (Boulier et Simon, 2010), les préservations des ressources végétales présentent un avantage certain puisqu'elles peuvent contribuer au développement de l'écotourisme au niveau local. 


\section{Conclusion} ficace de préservation du couvert végétal. Ainsi, en dépit des pressions que subissent les écosystèmes forestiers, les aires protégées ont permis de conserver une biodiversité importante. Cependant, dans certaines contrées, les pressions anthropiques ont eu raison des aires protégées. Dans ces cas de figure, les insuffisances sont à rechercher dans le mode de gestion et l'implication des populations locales.

\section{BIBLIOGRAPHIE}

AVAKOUDJO J., MAMA A., TOKO I., KINDOMIHOU V. \& SINSIN B. (2014), « Dynamique de l'occupation du sol dans le Parc National du W et sa périphérie au nord-ouest du Bénin ", Int. J. Biol. Chem. Sci, 8, 6, pp. 2608-2625.

BOULIER J., SIMON L. (2010), « Les forêts au secours de la planète : quel potentiel du stockage du carbone ? ", L'Espace géographique, 4, https://hal-paris1.archives-ouvertes.fr/hal-00590692/ document.

CIESLA W.M. (1997), Le Changement Climatique, les Forêts et l'Aménagement Forestier : Aspects Généraux, FAO-Organisation des Nations Unies pour l'alimentation et l'agriculture, Rome, http:// www.fao.org/docrep/v5240f/v5240f00.htm\#Contents.

DIMOBE K., OUÉDRAOGO A., SOMA S., GOETZE D., POREMBSKI S. \& THIOMBIANO A. (2015), "Identification of driving factors of land degradation and deforestation in the Wildlife Reserve of Bontioli (Burkina Faso, West Africa)", Global Ecology and Conservation, 4, pp. 559-571.

DONALD W.M., SPOONER J., DRESSING A.S. \& HARCUM J.B. (2011), "Statistical analysis for monotonic trends, Tech Notes 6. Developed for U.S.", Environmental Protection Agency by Tetra Tech, Inc., Fairfax, VA, 23 pp., www.bae.ncsu.edu/programs/extension/wqg/319monitoring/ tech_notes.htm. 
DIPAMA J.M. (2009), « Les risques de dégradation des écosystèmes liés à la culture du coton au Burkina Faso : le cas du parc national de Pô », Revue de l'Université de Moncton, 40, 2, pp. 29-52.

EMETERIO J.L.S., ALEXANDRE F., ANDRIEU J., GÉNIN A. \& MERING C. (2013), « Changements socioenvironnementaux et dynamiques des paysages ruraux le long du gradient bioclimatique nordsud dans le sud-ouest du Niger ", Vertigo - la revue électronique en sciences de l'environnement, 13, 3, http://vertigo.revues.org/14456.

FIASSON V. (1937), Rapport administratif de la mission dans le Parc de refuge du "W" du Niger, Niamey, $9 \mathrm{p}$.

FRATIANNI S., ACQUAOTTA F. (2010), « Les tendances et les indices de changement climatique des séries historiques dans le nord-ouest de l'Italie, $23^{\text {ème }}$ Colloque de l'Association Internationale de Climatologie, Rennes, Association Internationale de Climatologie, pp. 233-238.

GUINKO S. (1984), Végétation de la Haute Volta, Thèse de Doctorat ès Sciences naturelles, Univ. Bordeaux III.

GANSAONRÉ R.N. (2018), « Dynamique du couvert végétal et implications socioenvironnementales à la périphérie du Parc W/Burkina Faso, Vertigo - la revue électronique en sciences de l'environnement, 18, 1, http://journals.openedition.org/vertigo/20249.

GANSAONRÉ R.N., SODORE A.A. \& OUÉDRAOGO B. (in press), « Jeu des acteurs à la périphérie du Parc W du Burkina Faso : entre incoordination des interventions et faiblesses de moyens mobilisés », Afrique Science 16, 5, pp. 118-135, ISSN 1813-548X, http://www.afriquescience.net/ PDF/16/5/11.pdf.

HOUESSOU L.G., TEKA O., IMOROU I.T., LYKKE A.M. \& SINSIN B. (2013), "Land Use and Land-Cover Change at "W" Biosphere Reserve and Its Surroundings Areas in Benin Republic (West Africa)", Environment and Natural Resources Research, 3, 2, pp. 87-101.

HOUNTONDJI Y.C.H. (2008), Dynamique environnementale en zones sahélienne et soudanienne de l'Afrique de l'Ouest: Analyse des modifications et évaluation de la dégradation du couvert végétal, Thèse de Géographie, Faculté des Sciences, Université de Liège, 153 p.

INSTITUT NATIONAL DE LA STATISTIQUE ET DE LA DÉMOGRAPHIE (INSD) (2008), Recensement général de la population et de l'habitat de 2006, Ministère de l'Économie et des Finances, Burkina Faso, $86 \mathrm{p}$.

INSTITUT NATIONAL DE LA STATISTIQUE ET DE LA DÉMOGRAPHIE (INSD) (2000), Recensement général de la population et de l'habitat de 1996, Ministère de l'économie et des finances, Rapport final, Burkina Faso, 328 p.

KLEITZ G. (2001), Les zones périphériques des parcs du W (Bénin, Burkina Faso, Niger), Ecopas, CIRADEMVT, Montpellier, $68 \mathrm{p}$.

LOMPO O. (2002), La dynamique des acteurs à la périphérie du Parc W Burkina Faso. Rapport de recherche, ECOPAS, $85 \mathrm{p}$.

NACOULMA B.M.I. (2012), Dynamique et stratégie de conservation de la végétation et de la phytodiversité du complexe écologique du parc national du $W$ du Burkina Faso, Thèse de doctorat, Université de Ouagadougou, $202 \mathrm{p}$.

OUÉDRAOGO I., TIGABU M., SAVADOGO P., COMPAORÉ H., ODEN P.C. \& OUADBA J.M. (2010), "Land cover change and its relation with population dynamics in Burkina Faso, West Africa”, Land degradation and development, 21, pp. 453-462. 
OZER P., HOUNTONDJI Y.C., NIANG A.J., KARIMOUNE S., LAMINOU MANZO O. \& SALMON M. (2010), « Désertification au sahel : historique et perspectives », BSGLg, 54, pp. 69-84.

PONTIUS R.G. (2000), "Quantification error versus location error in comparison of categorical maps”, Photogrammetric engineering and remote sensing, 66, 8, pp. 1011-1016.

RASMUSSEN K., FENSHOLT R., FOG B., RASMUSSEN L.V. \& YANOGO I. (2014), “Explaining NDVI trends in northern Burkina Faso", Geografisk Tidsskrift Danish Journal of Geography, 114, 1, pp. 17-24, http://dx.doi.org/10.1080/00167223.2014.890522.

ROUDIER P. (2012), Climat et agriculture en Afrique de l'Ouest : Quantification de l'impact du changement climatique sur les rendements et évaluation de l'utilité des prévisions saisonnières, Thèse de doctorat, École des Hautes Études en Sciences Sociales (EHESS), France, 191 p.

SAWADOGO I. (2012), Ressources fourragères et représentations des éleveurs, évolution des pratiques pastorales en contexte d'aire protégée : Cas du terroir de Kotchari à la périphérie de la réserve de biosphère du W au Burkina Faso, Environnement et Société, Museum national d'histoire naturelle, 338 p.

SMITH L.C. (2000), “Trends in Russian Arctic river-ice formation and breakup, 1917 to 1994", Physical Geography, 20, 1, pp. 46-56.

SOULAMA S., KADEBA A., NACOULMA B.M.I., TRAORÉ S., BACHMANN Y. \& THIOMBIANO A. (2015), «Impact des activités anthropiques sur la dynamique de la végétation de la réserve partielle de faune de Pama et de ses périphéries (sud-est du Burkina Faso) dans un contexte de variabilité climatique », Journal of Applied Biosciences, 87, pp. 8047-8064.

TANKOANO B., HIEN M., DIBI N.H., SANON Z., AKPA Y.L., JOFACK SOKENG V.-C. \& SOMDA I. (2016), «Cartographie de la dynamique du couvert végétal du Parc National des Deux Balé à l'ouest du Burkina Faso », International Journal of Innovation and Applied Studies, 16, 4, pp. 837-846.

TSAYEM DEMAZE M. (2009), « Le protocole de Kyoto, le clivage Nord-Sud et le défi du développement durable ", L'Espace géographique, 38, 2, pp. 139-156.

VERMEULEN C. (2004), « Les enjeux de la gestion communautaire des grandes faunes : entre tension foncière et production cotonnière : le cas de Lara, périphérie du Parc W », Burkina Faso, Parcs et Réserves, 59, 4, pp. 20-27.

YANOGO P.I. (2011), Stratégies d'adaptation des populations aux changements climatiques autour du lac Bagré, Burkina Faso, Université d'Abomey Calavi Bénin, Thèse de Doctorat Unique de Géographie, $302 \mathrm{p}$.

ZOUNGRANA B.J.B. (2016), Vegetation dynamics in the southwest of Burkina Faso in response to rainfall variability and land use, Phd, Kwame Nkrumah University of Science and Technology, Kumasi, $162 \mathrm{p}$.

ZOUNGRANA B.J.B., CONRAD C., AMEKUDZI L.K., THIEL M., DA E.D., FORKUOR G. \& LÖW F. (2015), "Multi-Temporal Landsat Images and Ancillary Data for Land Use/Cover Change LULCC Detection in the Southwest of Burkina Faso, West Africa", Remote Sensing, 7, 9, pp. 12076-12102, www.mdpi.com/journal/remotesensing.

\section{RÉSUMÉS}

L'objectif de cette étude est d'analyser la dynamique du couvert végétal du Parc $\mathrm{W}$ du Burkina Faso et sa périphérie à travers les images Landsat et NDVI. Les données utilisées sont des images Landsat de 1984, 1999 et 2015 de résolution 30 mètres et une série temporelle d'images MODIS NDVI-250 mètres de la période 2001-2015. 
Des données de références collectées à l'aide d'un GPS et des connaissances locales ont permis une classification supervisée des images Landsat à l'aide de l'algorithme de maximum de vraisemblance. Le test de Mann-Kendall a été appliqué à la série d'images NDVI pour évaluer la tendance évolutive de la végétation.

Les résultats montrent que dans la zone hors parc, le couvert végétal a connu une nette régression. Cette régression s'est faite au profit des champs dont les superficies ont plus que doublé, passant de $20,2 \%$ à $46,4 \%$ entre 1984 et 2015 . Ainsi, $88,1 \%, 48,3 \%$ et $52,2 \%$ des superficies respectives de Botou, Diapaga et Tansarga sont affectées par la dégradation du couvert végétal. Selon le test de Mann-Kendal, 16,82 \% de la zone d'étude ont une tendance négative significative de la végétation. Cette tendance est principalement localisée dans les zones à emprise agropastorale, puisque $7,88 \%$ de ces superficies sont localisées dans des champs.

The objective of this study is to analyse the dynamics of the vegetation cover of Burkina Faso's W Park and its periphery through Landsat and NDVI images. The data used are Landsat images from 1984, 1999 and 2015 with a resolution of 30 meters and a time series of MODIS NDVI-250 meters images from the period 2001-2015. Reference data collected using GPS and local knowledge allowed a supervised classification of the Landsat images with the maximum likelihood algorithm. The Mann-Kendall test was applied to the NDVI time series to assess the vegetation trend.

The results showed that in the area outside the park, the vegetation cover has clearly regressed. This regression was in favour of croplands whose area has more than doubled from $20.2 \%$ to 46.4\% between 1984 and 2015. Thus, vegetation cover declined in $88.1 \%, 48.3 \%$ and $52.2 \%$ of the areas of Botou, Diapaga and Tansarga respectively. According to the Mann-Kendal test, $16.82 \%$ of the study area has a significant negative vegetation trend. This trend is mainly localized in the agropastoral zones, since $7.88 \%$ of these areas are located in fields.

\section{INDEX}

Mots-clés : dégradation, couvert végétal, image Landsat, Parc W, Burkina Faso

Keywords : degradation, environment, Landsat image, Park W, Burkina Faso

\section{AUTEURS}

\section{R. NOËL GANSAONRÉ}

Centre Universitaire de Gaoua, Burkina Faso, gnjumeaux@hotmail.com

BENEWINDÉ J.-B. ZOUNGRANA

Université Joseph Ki-Zerbo, Ouagadougou, Burkina Faso, beneboscoh@gmail.com

\section{P. ISIDORE YANOGO}

Université Norbert Zongo, Koudougou, Burkina Faso, yanogoisi@gmail.com 